\begin{abstract}
Objectives: To evaluate the implementation of a regular Nursing Round as an educational strategy for workplace learning in an intensive care unit with a single room environment.

Research Design: A multiple methods design was used. Fifty-four Nursing Rounds were observed, and nurses $(\mathrm{n}=40)$ completed bespoke evaluative surveys. Structured observational data and open-ended survey responses were submitted to content analysis and descriptive statistics were used to analyse survey findings.

Results: Nursing Rounds involved a diverse range of participants, most frequently nurses. The content most frequently discussed was empirical clinical issues, and nurses decided on nursing care actions to address these issues. The most frequently observed outcome of Nursing Rounds was knowledge translation. Nursing Rounds were perceived to positively influence application of evidence in practice, identification of areas for practice improvement and ability to communicate clinical information. Two categories emerged from analysis of open-ended survey questions; (1) 'Positive learning environment', where nurses described Nursing Rounds as a social learning experience; and (2) 'Impediments to Nursing Rounds', including difficulty attending Nursing Rounds due to competing priorities.
\end{abstract}

Conclusion: Nursing Rounds enabled evidence-based learning that enhanced inter-disciplinary collaboration. Further investigation may be required to understand how to enable nurses to attend more frequently, and generate a more holistic, evidence-based discussion.

\title{
Keywords:
}

Education; Evidence-based practice; Intensive care; Nursing; Nursing education; Patient rounds; Professional development; Teaching rounds

\section{Implications for Clinical Practice}


- Implementing Nursing Rounds into practice is a way to formalise social learning in the workplace, especially in single-room ICUs

- Nursing Rounds may increase inter-disciplinary collaboration and should be inclusive of a range of health professionals, patients (where able) and family members

- Facilitating Nursing Rounds in practice requires careful consideration of the topic to ensure facilitators acknowledge and integrate new issues, including patient and family-centred considerations and ethical views, to demonstrate these types of knowledge are valued 


\section{INTRODUCTION}

In Australia, there has been an increase in the number of intensive care units (ICUs) undergoing refurbishment or development as part of new hospital construction. Throughout this process, the design of the ICU should incorporate input from a range of professionals with experience in health care facility design, and importantly, clinicians from across a range of health care professions. Other more current and comprehensive guidelines exist to assist with the design of ICUs (Thompson, 2012) and cover a range of issues including the physical layout of the ICU; clinical, administrative and family support zones; and patient care zones.

In the ICU, patient care zones are configured either as multi-bed areas or single rooms. In recent years there has been a move towards accommodating patients in single room environments because research suggests these are associated with improvements in infection rates (Teltsch et al., 2011), patient and family satisfaction (Cepeda, 2005, Chaudhury et al., 2005, Chaudhury et al., 2006, Harris et al., 2006), privacy and sleep quality (Gabor et al., 2003). As such, a greater proportion of single rooms are often incorporated into the design of new ICUs. There is, however, less known about the impact of single room environments on other workplace issues (Maguire et al., 2013) such as learning in the workplace (Walsh et al., 2006).

\section{LITERATURE REVIEW}

Health care professionals are required to keep pace with rapidly evolving knowledge base, develop skills and attitudes appropriate for good practice, foster learning in the workplace, and sustain public confidence (McKee and Eraut, 2012). Individual clinicians are on a learning trajectory, developing knowledge over time through participation in various jobs and roles (McKee and Eraut, 2012). The workplace is a rich source of learning which can support ICU nurses to keep pace with the rapid developments and changes in intensive care research and practice (Billett, 2002). When nurses are faced with clinical uncertainty that 
is associated with the complexities that arise when caring for critically ill patients, opportunities for learning arise. However, this learning requires augmentation or support. In the ICU, common augmentation activities include explanations from more experienced clinicians, guided learning, and mnemonics (Billett and Smith, 2014). Through learning in the workplace, nurses and other clinical colleagues share knowledge about practice, providing an important foundation from which evidence-based care can be delivered.

Workplace characteristics that make full use of the knowledge resources of its members and locate and use relevant knowledge from outside the group are considered desirable for developing professional identity (Eraut, 2012). Within health care, the social interaction that occurs within and between professions is fundamental to optimising care, addressing challenging problems in clinical practice and facilitating inter-professional learning (Gregory et al., 2014). Engagement with colleagues is important for maximising learning opportunities and single room environments can limit this type of engagement (Egan and Jaye, 2009). Social interaction is recognised as an important avenue through which nurses seek information with other people being identified as preferred sources of information when nurses are faced with clinical uncertainty (Marshall et al., 2011). Frequently this social interaction, which leads to learning in the workplace, is spontaneous but can also be planned. The interaction and engagement that occurs during social interaction of health professionals is varied and dependent on the need for learning and the space in which this learning occurs (Gregory et al., 2014).

In the ICU, informal and incidental workplace learning may occur when nurses observe and hear what is happening in nearby patient care areas. The multi-bed ICU is an open space, where observation and listening is commonplace; it is a space that enables learning (Gregory et al., 2014). It has been common practice in multi-bed ICUs to allocate patients by alternating junior and more senior nurses and it has been reported that the self- 
reported competence of less experienced ICU nurses is correlated with learning from others (Takase et al., 2015). In multi-bed ICUs, through observation of more experienced nurses, nurses learn how they manage patients of increased complexity. The single room environment presents a challenge to workplace learning because nurses are isolated in a single room and cannot necessarily see or hear what nurses are doing and saying in other bed spaces. Thereby the single room environment constrains learning (Gregory et al., 2014).

Nursing Rounds is a practice pedagogy that has been used to promote workplace learning for nurses. Nursing Rounds have been explored in surgical (Gardner et al., 2010) and emergency (Dalmaso et al., 2015) settings. Participants in Nursing Rounds reported the potential to improve clinical practice, enhance collaboration and 'refresh' forgotten information (Dalmaso et al., 2015). Given the reduced opportunities for peer observation and learning associated with the single room environment, Nursing Rounds was implemented in one ICU as a way to augment nurses' learning from their work.

\section{OBJECTIVES}

The aim of this study was to evaluate the implementation of a regular Nursing Round as an educational strategy for workplace learning in an intensive care unit with a single room environment.

\section{METHODS}

\section{$\underline{\text { Study design }}$}

A multiple methods design was used to evaluate the implementation of Nursing Rounds for workplace learning in the ICU setting. Phase 1 involved observations of Nursing Rounds, and Phase 2 involved nursing participant surveys.

\section{$\underline{\text { Structure of Nursing Rounds }}$}


Nursing Rounds were held for a one-hour period, usually twice each week. Prior to commencing, two patients were identified by senior nursing staff for involvement in Nursing Rounds; patients (and their clinical issues) were often selected based on current team learning needs. Nursing Rounds were facilitated by the Clinical Nurse Consultant (CNC) or delegate and were supported by a Nursing Professor. Nursing Rounds participants included multidisciplinary health care professionals, research staff, patients and family and industry colleagues.

The structure of Nursing Rounds took place as per Figure 1. On commencement of Nursing Rounds, the patient's primary nurse, with support from the Clinical Nurse Consultant (CNC), presented a face-to-face summary of the patient's history and presenting problem at the patient's bedside, which was used to develop a clinical question(s) by members of the group. A clinical question was defined as a matter requiring resolution or discussion that was specific to the clinical care of the patient. The facilitator CNC then stimulated group discussion, to explore the clinical question(s). Nurses engaged in discussions about different sources of information, weighing these different sources of evidence, and drew conclusions about the best decision. This resulted in nurses deciding on the best possible actions that should be undertaken for the patient.

\section{$\underline{\text { Setting }}$}

This study was undertaken at one health service in southeast Queensland, Australia. The ICU was a Level 3 unit, with 17 funded beds where the percentages of nursing staff with critical care qualifications was 53\%. The nurse-to-patient ratio was 1:1 for ventilated patients and 1:2 for stable non-ventilated patients. An ACCESS nurse was available to provide "on-thefloor" Assistance, Coordination, Contingency, Education, Supervision and Support (Australian College of Critical Care Nurses, 2003). There was 1 ACCESS nurse for every four beds. 
$\underline{\text { Participants }}$

Participants in the observations included all those present at Nursing Rounds; including health care professionals, research staff, patients and family. For surveys, all registered nurses $(n=110)$ employed full- or part-time in the ICU were invited to participate during the study period.

\section{$\underline{\text { Data collection }}$}

Phase one observations: Nursing Rounds were observed, using a specifically prepared document. The semi-structured data collection form included tables to document; 1) the number and role of participants; 2) all clinical questions formulated for the patient (addresses 'formulate clinical question' in Figure 1); and 3) the decisions and actions decided on to overcome the identified care issues (addressees 'make patient care decision and take action' in Figure 1.). One researcher was present at each round to collect data (AM, NM). Owing to the iterative nature of the discussion during Nursing Rounds, where the clinical question(s) were explored and nurses used different sources of information to draw conclusions about the best decision, data relating to 'identify information and alternatives and weigh evidence' as per Figure 1 were not explicitly collected. Usually, part of the decision-making process is evaluating outcomes of the decision, such as patient outcomes (see Figure 1) (Dowding and Thompson, 2009), however, we did not undertake this. Instead we observed the interactions and discussion among nurses and other health professionals taking part in this formalised information sharing encounter. Observations allowed the researchers to see how health care professionals adhered to the implementation of Nursing Rounds, facilitating evaluation of the implementation of Nursing Rounds.

Phase two surveys: Nurses completed bespoke surveys which explored their experience of the Nursing Rounds as an educational strategy. Survey questions used Likert-type items 
ranging from 1 to 5 , with 1 indicating strongly disagree and 5 indicating strongly agree. All Likert-type items provided the opportunity for open-ended responses. Four questions had no Likert rating, but instead provided space for nurses to provide open-ended responses, these included

1. 'What are the perceived benefits, if any, of Nursing Rounds?';

2. 'What helped and hindered your ability to participate in Nursing Rounds?';

3. 'Did you present a patient in Nursing Rounds or facilitate a Nursing Rounds? With prompts including:

a. Did you feel supported in the process?

b. Was it a positive learning experience?

c. Did it change the way in which you might present clinical information to medical and allied health team members?'; and

4. 'Do you have any suggestions on how Nursing Rounds could be done differently?'.

Demographic data including age, initial and highest nursing qualifications, highest educational qualifications, experience in nursing, experience as a critical care nurse and fraction of full time employment were collected from survey participants. Surveys were administered in-person by one researcher (LH).

\section{Ethical considerations}

The study was approved by the Human Research Ethics Committee of the health service and local university. Written consent was obtained at the time of observations. Consent was implied by completing and returning surveys. Where relevant, assent was obtained to collect information during Nursing Rounds from patients and/or family members.

\section{Data analysis}


Phase one observations: All observational data were entered into Microsoft Excel (2016) for data management. Three researchers independently coded data using content analysis (AM, GT, LG). Entries were coded line-by-line, based on the broader focus on the type of information/knowledge discussed (i.e. the observation note "Systolic blood pressure $<100$, potential haemodynamic compromise", was coded as assessment of patient data, rather than focussing more narrowly on blood pressure). Data coded as similar content were given higher level category labels. The researchers met to question and confirm findings, deciding on final analysis reported in the manuscript.

Phase two surveys: Responses to Likert-type items and demographic data from surveys were analysed using descriptive statistics. Participant characteristics were reported with median and interquartile ranges for continuous data, as the data were not normally distributed. Categorical data were presented with absolute and relative frequencies. Likerttype responses were reported using median and interquartile range (IQR). Questions with Likert-type responses also had the options of adding comments, which were used to understand participants' ratings. The four open-ended questions were analysed using inductive content analysis independently by two researchers (GT, LH) (Elo and Kyngäs, 2008). The responses for the four questions were entered into Microsoft Excel (2016) to assist with data management. Responses were read through several times to gain a sense of the whole. Each response was coded with a word or sentence that described the content of the response. In Microsoft Excel (2016), codes that belonged together, were grouped to form subcategories. Subcategories were then examined for similarities and differences, allowing higher order categories to be developed. These finding were questioned and confirmed by the research team.

\section{FINDINGS}


The findings from the two phases are presented separately. First the observational data are presented including the observed characteristics of Nursing Rounds, such as participants and frequency, followed by analysis of the observed content of Nursing Rounds including the clinical question, actions selected and outcomes. Second, Phase 2 findings are presented, including characteristics of survey respondents, analysis of Likert-type response questions and analysis of open ended questions.

Phase one observations Over a period of 12 months, from June 2014 to May 2015, approximately 60 Nursing Rounds occurred and 54 were observed and field notes were documented. A median of ten (range 5-20) Registered Nurses (inclusive of nurses with clinical, education, leadership and research roles) participated in each of the Nursing Rounds. Other participants included medical $(n=3)$ and allied health ICU staff (dietitian $(n=5)$, pharmacist $(n=1)$, physiotherapist $(n=1)$, nurse specialists from other clinical areas $(n=10)$, nursing students $(n=4)$, family members $(n=3)$, and medical device representative $(n=1)$.

Our analysis of observations showed the sessions followed a pattern (see Table 1). Initially participants discussed the clinical issue. A total of 117 issues were identified (median 2; range 1-3) were discussed per Nursing Round. Actions were decided for each clinical issue. Most issues identified $(\mathrm{n}=84)$ were empirical in nature and related to patient assessment and physiology such as "abdominal sepsis". Other issues included aspects of care coordination $(n=11)$ or were equipment related $(n=23)$. Next, nurses discussed what could be done for the patient, outlining a total of 154 nurse practices required, for the previous quote, the actions included "1) look for source of sepsis, 2) remove all lines to exclude possible sources". Nurses also frequently discussed multi-disciplinary collaborative actions required such as "Ask dietitian to review”. Nurses discussed patient-centred and ethical actions less frequently. 
As a result of Nursing Rounds other events which were not patient related were observed during data collection, of which knowledge translation was the most frequent. These events were sometimes triggered by members of the Nursing Rounds having queries about the best evidence and having to follow up this knowledge deficit. For instance, nurses were unsure about steroid use during sepsis, which was investigated during Nursing Rounds: "Evidence based practice for steroid use in sepsis: discussed steroid use should only be used in shock phase of sepsis- outlined steroids have supportive and negative research for their use”. However, observers noted that participants sometimes spoke about the body of evidence, but did not explicitly refer to sources other than participants present at the Nursing Rounds.

(Table 1 here).

Phase two surveys

Forty ICU nursing staff completed surveys, a 36.4\% response rate. The median age of nurse participants was 34 years, and most nurses were Level 1 Registered Nurses, who had undertaken their Bachelor Degree and were employed part-time (Table 2). Of the 40 respondents, three had not previously attended Nursing Rounds but were aware of this activity.

(Table 2 here).

Our analysis of survey data showed responses to Likert-type items related to the usefulness of Nursing Rounds and some perceived improvements scored highly. Against the 5-point Likert-type items nurses reported a willingness to attend Nursing Rounds (Median=5, $\mathrm{IQR}=5$ ), viewed Nursing Rounds as a useful education strategy (Median=5, IQR=4-5), and valued interdisciplinary involvement (Median=5, IQR=4-5). Those providing additional open-ended responses for these questions revealed Nursing Rounds were beneficial as an 
adjunct to other educational strategies $(n=19)$. There was agreement that Nursing Rounds helped identify areas for practice improvement, closed the evidence-practice gap and enhanced clinical communication (See Table 3).

(Table 3 here).

Some responses were scored lower. Nurses' responses on the influence of Nursing Rounds on team work were neutral (see Table 3), with some nurses adding comments that they were unsure $(n=7)$, that teamwork may have been enhanced only during the Nursing Round $(n=5)$, or that teamwork was already strong on the unit $(n=3)$. There were neutral responses to the influence of Nursing Rounds on confidence (See Table 3). Additional openended comments for self-confidence revealed six nurses thought Nursing Rounds improved their confidence, while six nurses avoided presenting at rounds or did not have the opportunity to attend rounds.

For the four open-ended questions, inductive content analysis allowed researchers to identify two categories, including 'impediments to Nursing Rounds' and 'positive learning environment'.

\section{Category 1: Impediments to Nursing Rounds}

Nurses described factors which prevented their ability to participate in Nursing Rounds including the timing of Nursing Rounds and an inability to attend Nursing Rounds. Nurses stated that Nursing Rounds were scheduled for the afternoon, which facilitated afternoon shift nurse attendance; however, nurses rostered for day shifts lacked opportunities to participate in Nursing Rounds. One nurse expressed: 'Timing- because of 8-hour shifts and handover time crossed over with it, I would only get to go if I was on a late. NI'. Further, bedside nurses and ACCESS nurses found it difficult to attend as they were unable to leave their patients, and they often experienced having no staff to cover their patient if they were to attend Nursing Rounds. For example, a nurse stated: 'Being a bedside nurse and having a 
patient to look after - not being able to leave. N2'. Furthermore, nurses portrayed a busy environment, where competing patient and non-patient tasks were prioritised over Nursing Rounds attendance.

There were mixed views on the required frequency of Nursing Rounds. Ten nurses thought the frequency of Nursing Rounds was sufficient, while 13 nurses stated they needed to be increased, with three nurses suggesting having rounds at different times to increase chances of being able to attend.

\section{Category 2: Positive learning environment}

In open-ended responses, nurses described Nursing Rounds as an opportunity to expand their knowledge. Nurses liked the content of Nursing Rounds, describing it as high quality, relevant to nursing and thought-provoking. Nurses wanted evidence-based information, and believed that the facilitator and the amount of preparation prior to a Nursing Round were influencing factors.

The learning process was viewed as engaging, as nurses had the opportunity to freely and safely ask questions, and actively contribute to the nursing or interdisciplinary discussion: 'Promoted engaging and informative discussions in an environment that promotes learning for all skill levels. N3'. Of the 40 participants, 13 reported that they had introduced and presented patient information at the beginning of a Nursing Round and most agreed that they felt supported $(n=11)$ and had a positive learning experience $(n=9)$.

Finally, Nursing Rounds were viewed as a chance for exposure for new and different patient cases. It was seen as beneficial for both junior and senior nurses to visualise and learn about different patient cases which they either had not been exposed to before, or for more senior nurses, a refresher on a patient and diagnosis they had not managed recently: 
As a group it exposes a group to learning about a disease or equipment or an intervention. It has us as a group consider things we might not of [sic] known. It's good to have a refresher if you have come from other units etc. It helps novice nurses consolidate learning. Group environment shows them it's safe to ask questions. N4 


\section{$\underline{\text { DISCUSSION }}$}

In this study, we found Nursing Rounds was targeted at, and attended by, nurses but observations of practice and nurses' perceptions revealed it enabled interdisciplinary collaboration. The content of Nursing Rounds tended to focus on empirical clinical issues, and nurses addressed these by doing nursing care, or collaborating with the inter-disciplinary team. Nurses' perceptions of Nursing Rounds showed it was a positive learning experience and was useful, but there were barriers such as inability to attend Nursing Rounds. Observations of Nursing Rounds and nurses' perceptions of Nursing Rounds highlighted that it can enable knowledge translation; however sources of evidence were often limited to the facilitator as expert.

We found Nursing Rounds were a pedagogic activity that could potentially promote learning. Participants described Nursing Rounds as a positive, useful and engaging experience, demonstrating a 'community of practice' where people engaged in shared social learning in the workplace (Wegner-Trayner and Wegner-Trayner, 2015). Nurses' views of Nursing Rounds as a positive experience is perhaps unsurprising, given nurses are not passive learners; they engage in dynamic processes and are participatory leaners (Armola et al., 2010). Although we did not test learning outcomes, learning between nurses has been shown to improve post implementation of Nursing Rounds (Aitken et al., 2011, Gardner et al., 2010). Nursing Rounds formalises social learning (Estabrooks et al., 2005), as it addresses the loss of implicit communities of practice, which exist in ICUs with an open plan, when single rooms are introduced.

For this pedagogic activity to be sustained in practice, Nursing Rounds facilitators may need to address impediments identified. For instance, the frequency 
of Nursing Rounds may need to be considered. Like our findings, Aitken et al., (2011) found nurses had mixed views on the optimal frequency of Nursing Rounds. Additionally, like previous work, some nurses were unable to attend Nursing Rounds as priority was given to clinical care (Nicolini et al., 2008). Researchers have reported nurses' feelings of being 'tethered' to spaces due to patient acuity, which limits their ability to undertake information-seeking encounters (Spenceley et al., 2008).

Activities nurses undertake varies between settings, in ICU it has been found that $5 \%$ of nursing time is unit-related activities like education (Abbey et al., 2012), while in the ward setting $14.5-30.3 \%$ of time is spent on unit-related activities like education (van den Oetelaar et al., 2018). Yet, nurses in both settings spend similar amounts of time providing direct patient care (Abbey et al., 2012, van den Oetelaar et al., 2018). Thus, strategies may need to be explored to find a way for ICU patients to continue to receive 1:1 care during Nursing Rounds, so ICU nurses can leave rooms for non-direct patient care activities. At shift changes, nurses from both shifts are present and may present an ideal opportunity for some staff to attend while others stay with patients. Overall, when organising social learning events, the organiser needs to consider the ever-changing context, to determine when and how often Nursing Rounds need to occur (Wenger, 1998).

As we continue to promote work-based learning to improve practice in the workplace, there are issues for nurses interested in counting these learning experiences towards their continuing professional development (CPD) requirements. As a component of annual registration (Nursing And Midwifery Board of Australia, 2016), the Australian Health Practitioner Regulation Agency (AHPRA) requires evidence that registered nurses participate in continuing professional development activities. Thus, formalising Nursing Rounds would be necessary to allow nurses to 
get CPD credit. Counting Nursing Round attendance towards CPD credit may assist nurses to meet regulatory requirements and provide motivation for attending these rounds.

Even though we purposefully focused on nursing decisions and learning during Nursing Rounds, it heightened communication between disciplines, settings and groups. In our study, membership in Nursing Rounds was largely Registered Nurses within a single ICU, however, we found that other health professionals and healthcare researchers were sometimes present. Having a diverse range of health care professionals together, may have enabled brokering of knowledge between communities, although this outcome was not tested in our study (Wenger, 1998), as each discipline can introduce elements from their own practice. The presence of interdisciplinary staff at Nursing Rounds is consistent with reports from studies of Nursing Grand Rounds (NGRs), which are presentations given by nurses at venues often away from the bedside (Armola et al., 2010). Researchers report other health care professionals' interest in attending NGRs was an unexpected outcome, and increased inter-professionalism (Laibhen-Parkes et al., 2015). Although it may not be feasible for all disciplines to attend Nursing Rounds, their involvement should be encouraged to promote sharing of knowledge across groups. Alternatively, other health care professionals could implement their own bedside rounds, to foster inter-disciplinary learning on discipline specific topics.

In our study Nursing Rounds also promoted nurses from other settings to take part, which has been reported as a collaborative and integrative experience (Jennings, 2017). Additionally, we found others like families and medical device representatives were present at Nursing Rounds. This finding was supported in the NGRs literature, as NGRS have been shown to enhance patient and family engagement (Armola et al., 
2010, Dalmaso et al., 2015). Given the paucity of research on family involvement in Nursing Rounds, future studies should investigate family members' perceptions of involvement in this activity.

The focus of Nursing Rounds tended to be on empirical facts about medically related care, which may have overshadowed other types of learning. Like our study, the focus of Nursing Rounds tends to be discussing patient condition, treatment and associated monitoring (Dalmaso et al., 2015), which tended to have a medical focus. The actions decided by nurses in our study are similar to Aitken et al.'s (2011) work, where nurses made decisions about physical nursing care and practice, or to consult with inter-disciplinary health care professional. Again like Aitken et al.'s (2011) work, discussions that considered the patient in the context of family, or that were ethical in nature were less common. A facilitator's guide to Nursing Rounds, noting the patterns of knowing in nursing (White, 1995), could support discussion of empirical, procedural, ethical, personal and socio-political knowledge and understanding during each round.

In our study, nurses respected and valued Nursing Rounds facilitators to support learning. Nurses' perceptions and our observations of Nursing Rounds suggested translation of evidence into practice occurred via a facilitator. People are the most common and preferred source of information for nurses, and nurses will often look to senior clinicians in close proximity when they require 'high quality' information (Marshall et al., 2011). This is often because nursing peers are perceived to address immediate care needs, give unique support and have relevant experience in that setting (Estabrooks et al., 2005). David Hume ((1977 [1748])) and Alvin Goldman (2001) have done seminal work on the epistemology of testimony, and it may be that Nursing Rounds participants valued facilitators because they had 
experienced their track record of providing accurate information, and they presented it in an appropriate manner (Fallis, 2004). The role of the ACCESS nurse in supporting clinical education in the ICU is important. In the context of Nursing Rounds, the ACCESS nurse could be used as a facilitator in Nursing Rounds or to relieve bedside nurses from clinical care, enabling them to participate in the learning activity.

Nursing facilitators are a legitimate source of evidence (Estabrooks et al., 2005), however, there are a number of factors that participants in Nursing Rounds need to consider when using testimony from the facilitator. For instance, participants should consider the plausibility of the information based on their prior knowledge and experiences (Fallis, 2004). Further, the receiver should use other sources of evidence to corroborate the facilitator's testimony (Fallis, 2004), which was not witnessed during Nursing Rounds. However, verifying information can be time-intensive and costly (Fallis, 2004). In one study, a librarian was present during Nursing Rounds (Aitken et al., 2011); a strategy to enhance information verification. Researchers have shown that nurses often do not verify information against other sources like published research because the large volume is daunting, it is not accessible and nurses lack skills to critique it (Marshall et al., 2011). Thus, enabling nurses' capabilities and opportunities to use other sources of evidence, such as computerised access at each bed to access policies, guidelines, research, is an important consideration for future implementers of Nursing Rounds.

\section{LIMITATIONS}

This study was confined to a single ICU and as such, the suitability of Nursing Rounds in other settings would need to be evaluated. We had a small response rate to the surveys, and most respondents had not led a Nursing Round, which could influence their responses. In our observations we did not capture the way nurses 
weighted their options before deciding on actions, this could be the focus on future work, and may require different methods.

\section{CONCLUSION}

In our study, Nursing Rounds may have formalised social workplace learning. While continuing professional development opportunities are often conceptualised as formal education such as workshops, hospital based courses and academic programs, workplace learning is also an important component of learning. The Nursing Rounds process appeared to enable a more multi-disciplinary approach to care and translation of knowledge into practice. However, future users of Nursing Rounds may consider how to facilitate a more holistic discussion, how to enhance nurse attendance and how to create opportunities to use more than one source of evidence. Our study highlights the importance of nursing leaders within the ICU who organise and facilitate Nursing Rounds, as they play an important role in establishing conducive learning environments for staff. 


\section{$\underline{\text { REFERENCES }}$}

Abbey, M., Chaboyer, W., Mitchell, M., 2012. Understanding the work of intensive care nurses: A time and motion study. Aust Crit Care. 25, 13-22.

Aitken, L.M., Burmeister, E., Clayton, S., Dalais, C., Gardner, G., 2011. The Impact of Nursing Rounds on the practice environment and nurse satisfaction in intensive care: Pre-test post-test comparative study. Int J Nurs Stud. 48, 918-925.

Armola, R.R., Brandeburg, J., Tucker, D., 2010. A guide to developing Nursing Grand Rounds. Crit Care Nurse. 30, 55-62.

Australian College of Critical Care Nurses, 2003. ACCCN ICU staffing position statement (2003) on Intensive Care Nursing Staffing. https://www.accen.com.au/documents/item/20 (accessed 28.03.18).

Billett, S., 2002. Toward a workplace pedagogy: Guidance, participation and engagement. Adult Education Quarterly. 53, 27-43.

Billett, S., Smith, R., 2014. Learning in the circumstances of professional practice, in: Billett S., Harteis C., Gruber H. (Eds.), International Handbook of Research in Professional and Practice-based Learning. Springer, Dordrecht, pp. 733757.

Cepeda, J.A., Whitehouse, T., Cooper, B.,Hails, J., Jones, K., Kwaku, F., Taylor, L., Hayman, S., Cookson, B., Shaw, S., Kibbler, C., Singer, M., Bellingan, G. \& Wilston, P.R., 2005. Isolation of patients in single rooms or cohorts to reduce spread of MRSA in intensive care units:prospective two-centre study. Lancet. 2005, 295-304.

Chaudhury, H., Mahmood, A., Valente, M., 2005. Advantages and disadvantages of single-versus multiple-occupancy rooms in acute care environments. Environ Behav. 37, 760-786. 
Chaudhury, H., Mahmood, A., Valente, M., 2006. Nurses' perception of single-occupancy versus multioccupancy rooms in acute care environments: An exploratory comparative assessment. Appl Nurs Res. 19, 118-125.

Dalmaso, K., Weber, S., Eley, R., Spencer, L., Cabilan, C.J., 2015. Nurses' perceived benefits of trauma nursing rounds (TNR) on clinical practice in an Australian emergency department: A mixed methods study. Australasian Emergency Nursing Journal. 18, 42-48.

Dowding, D., Thompson, C.D., 2009. Essential Decision Making and Clinical Judgement for Nurses. Churchill Livingstone, New York.

Egan, T., Jaye, C., 2009. Communities of clinical practice: The social organization of clinical learning. Health. 13, 107-125.

Elo, S., Kyngäs, H., 2008. The qualitative content analysis process. J Adv Nurs. 62, 107-115.

Eraut, M., 2012. Developing a broader approach to professional learning, in: McKee A.E.M. (Eds.), Learning Trajectories, Innovation and Identity for Professional Development. Springer, Netherlands, pp. 21-45.

Estabrooks, C.A., Rutakumwa, W., O’Leary, K.A., Profetto-McGrath, J., Milner, M., Levers, M.J., et al., 2005. Sources of practice knowledge among nurses. Qual Health Res. 15, 460-476.

Fallis, D., 2004. On verifying the accuracy of information: Philosophical perspectives. Library Trends. 52, 463-487.

Gabor, J.Y., Cooper, A.B., Crombach, S.A., Lee, B., Kadikar, N., Bettger, H.E., et al., 2003. Contribution of the intensive care unit environments to sleep disruption in mechanically ventilated patients and healthy subjects. Am J Respir Crit Care Med. 167, $708-715$. 
Gardner, G., Woollett, K., Daly, N., Richardson, B., Aitken, L.M., 2010.

Innovation in clinical learning for the acute hospital environment: Nursing Grand Rounds. Nurse Educ Today. 30, 737-741.

Goldman, A.I., 2001. Experts: Which ones should you trust? Philosophy and phenomenological research. 63, 85-110.

Gregory, L.R., Hopwood, N., Boud, D., 2014. Interprofessional learning at work: What spatial theory can tell us about workplace learning in an acute care ward. Journal Of Interprofessional Care. 28, 200-205.

Harris, D.D., Shepley, M.M., White, R.D., Kolberg, K.J.S., Harrell, J.W., 2006. The impact of single family room design on patients and caregivers: Executive summary. J Perinatol. 26, 38-48.

Hume, D., (1977 [1748]). An Enquiry Concerning Human Understanding. Hackett, Indianapolis.

Jennings, F.L., 2017. Intensive care nurses' perceptions of Inter Specialty Trauma Nursing Rounds to improve trauma patient care--A quality improvement project. Intensive Crit Care Nurs. 40, 35-43.

Laibhen-Parkes, N., Brasch, J., Gioncardi, L., 2015. Nursing Grand Rounds: A strategy for promoting evidence-based learning among pediatric nurses. J Pediatr Nurs. 30, 338-345.

Maguire, D.J., Burger, K.J., O'Donnell, P.A., Parnell, L., 2013. Clinician perceptions of a changing hospital environment. Health Environments Research \& Design Journal. 6, 69.

Marshall, A.P., West, S.H., Aitken, L.M., 2011. Preferred information sources for clinical decision making: Critical care nurses' perceptions of information accessibility and usefulness. Worldviews Evid Based Nurs. 8, 224-235. 
McKee, A., Eraut, M, 2012. Introduction. In: McKee A.E., (Eds.), Learning Trajectories, Innovation and Identity for Professional Development. Netherlands: Springer, pp 1-19.

Nicolini, D., Powell, J., Conville, P., Martinez - Solano, L., 2008. Managing knowledge in the healthcare sector. A review. International Journal of Management Reviews. 10, 245-263.

Nursing And Midwifery Board Of Australia, 2016. Registration standard: Continuing professional development 2016. http://www.nursingmidwiferyboard.gov.au/Registration-Standards/Continuingprofessional-development.aspx (accessed 12.02.18).

Spenceley, S.M., O'Leary, K.A., Chizawsky, L.L.K., Ross, A.J., Estabrooks, C.A., 2008. Sources of information used by nurses to inform practice: An integrative review. Int J Nurs Stud. 45, 954-970.

Takase, M., Yamamoto, M., Sato, Y., Niitani, M., Uemura, C., 2015. The relationship between workplace learning and midwives' and nurses' self-reported competence: A cross sectional survey. Int J Nurs Stud. 52, 1804-1815.

Teltsch, D.Y., Hanley, J., Loo, V., Goldberg, P., Gursahaney, A., Buckeridge, D.L., 2011. Infection acquisition following intensive care unit room privatization. Arch Intern Med. 171, 32-38.

Thompson, D.R., Hamilton, E.K., Cadenhead, C.D., Swoboda, S.M., Schwindel, S.M., Anderson, D.C., Schmitz, E.V., St Andre, A.C., Axon, D.C., Harrell, J.W., Harvey, M.A., Howard, A., Kaufman, D.C. \& Petersen, C. , 2012. Guidelines for intensive care unit design. Crit Care Med. 2012, 1586-1600. 
van den Oetelaar, W.F.J.M., van Stel, H.F., van Rhenen, W., Stellato, R.K., Grolman, W., 2018. Mapping nurses' activities in surgical hospital wards: A time study. PLoS One. 13, e0191807.

Walsh, W.F., McCullough, K.L., White, R.D., 2006. Room for improvement: Nurses' perceptions of providing care in a single room newborn intensive care setting. Adv Neonatal Care. 6, 261-270.

Wegner-Trayner, E., Wegner-Trayner, B., 2015. Introduction to communities of practice: A brief overview of the concept and its uses. http://wengertrayner.com/introduction-to-communities-of-practice/ (accessed 21.07.17).

Wenger, E., 1998. Communities of Practice: Learning, Meaning, and Identity. Cambridge University Press, Cambridge.

White, J., 1995. Patterns of knowing: review, critique, and update. Advances in Nursing Science. 17, 73-86. 